\title{
Cooperative Demonstrable Data Retention for Integrity Verification in Multi-Cloud Storage
}

\author{
Krishna Kumar Singh \\ GIDA Gorakhpur \\ U.P India
}

\author{
Rajkumar Gaura \\ GIDA Gorakhpur \\ U.P India
}

\author{
Sudhir Kumar Singh \\ GIDA Gorakhpur \\ U.P India
}

\begin{abstract}
Demonstrable data retention (DDR) is a technique which certain the integrity of data in storage outsourcing. In this paper we propose an efficient DDR protocol that prevent attacker in gaining information from multiple cloud storage node. Our technique is for distributed cloud storage and support the scalability of services and data migration. This technique Cooperative store and maintain the client's data on multi cloud storage. To insure the security of our technique we use zero-knowledge proof system, which satisfies zero-knowledge properties, knowledge soundness and completeness. We present a Cooperative DDR (CDDR) protocol based on hash index hierarchy and homomorphic verification response. In order to optimize the performance of our technique we use a novel technique for selecting optimal parameter values to reduce the storage overhead and computation costs of client for service providers.
\end{abstract}

Keyword: Demonstrable Data Retention, homomorphic, zero knowledge, storage outsourcing, multiple cloud, Cooperative, data Retention.

\section{INTRODUCTION}

IN past few years, a cloud storage service has become a faster profitable growth point by providing their clients a reasonably scalable, low-cost, position-independent platform for client's data. As cloud computing environment is made based on open architectures and interfaces, it has the capability to incorporate multiple internal or/and external cloud services together to provide high interoperability. We say such a distributed cloud environment as a hybrid cloud (or multi-Cloud). Very often, we use virtual infrastructure management (VIM) [2], a multi-cloud allows clients to easily access his or her resources remotely through interfaces such as Web services provided by Amazon EC2. There exist various tools and technologies for multicloud, such as Vmware vSphere, Platform VM Orchestrator and Ovirt. These tools help cloud providers to construct a distributed cloud storage platform (DCSP) for managing client's data. However, such an important platform is vulnerable to be compromised, especially in a hostile environment and it would bring irretrievable losses to the clients. For examplethe confidential data in an enterprise may be illegally accessed through a remote interface provided by a multi-cloud, or confidential data and archives may be lost or altered with when they are stored into a hostile storage pool outside the enterprise. Therefore, it is important and necessary for cloud service providers (CSPs) to provide security techniques for managing their storage services. Demonstrable data retention (DDR) [1] (or proofs of retrievability (POR) [2]) is such a probabilistic proof technique for a storage provider to prove the integrity and ownership of clients' data without downloading data. The verification without downloading makes it especially important for large-size files and folders (typically including many clients' files) to check whether these data have been altered with or deleted without downloading the latest version of data. Thus, it is able to replace traditional hash and signature functions in storage outsourcing. Various DDR techniques have been recently proposed, such as Scalable DDR [4] and Dynamic DDR [5]. However, these techniques mainly focus on DDR issues atuntrusted servers in a single cloud storage providerand are not suitable for a multi-cloud environment (see the comparison of POR/DDR techniques in Table 1)Motivation: In order to provide a low-cost, scalable, location-independent platform for managing clients' data, current cloud storage systems adopt several new distributedfile systems, for example, Google File System (GFS),Apache Hadoop Distribution File System (HDFS), Amazon S3 File System, CloudStore etc. These file systems share 
International Journal of Computer Applications Technology and Research

Volume 2- Issue 2, 195 - 207, 2013, ISSN: 2319-8656

TABLE 1: Comparison of POR/DDR schemes for a file consisting of $n$ blocks

\begin{tabular}{|c|c|c|c|c|c|c|c|c|}
\hline Scheme & Type & $\begin{array}{l}\text { CSP } \\
\text { Comp. }\end{array}$ & $\begin{array}{l}\text { Client } \\
\text { Comp. }\end{array}$ & Comm. & Flag. & $\begin{array}{l}\text { Priva } \\
\text { cy }\end{array}$ & $\begin{array}{l}\text { Multiple } \\
\text { Clouds }\end{array}$ & $\begin{array}{l}\text { Prob. Of } \\
\text { Detection }\end{array}$ \\
\hline DDR[2] & HomT & $\mathrm{O}(\mathrm{t})$ & $\mathrm{O}(\mathrm{t})$ & $\mathrm{O}(1)$ & & $\checkmark$ & \# & $1-(1-\rho) t$ \\
\hline SDDR[4] & MHT & $\mathrm{O}(\mathrm{t})$ & $\mathrm{O}(\mathrm{t})$ & $\mathrm{O}(\mathrm{t})$ & $\checkmark$ & $\checkmark$ & & $1-(1-\rho) t \cdot s$ \\
\hline CDDR-[5] & MHT & $\mathrm{O}(\mathrm{t} \cdot \log \mathrm{n})$ & $\mathrm{O}(\mathrm{t} \cdot \log \mathrm{n})$ & $\mathrm{O}(\mathrm{t} \log \mathrm{n})$ & & $\checkmark$ & & $1-(1-\rho) t$ \\
\hline CDDR-II[5] & MHT & $\mathrm{O}(\mathrm{t} \log \mathrm{n})$ & $\mathrm{O}(\mathrm{t} \log \mathrm{n})$ & $\mathrm{O}(\mathrm{t} \log \mathrm{n})$ & & & & $1-(1-\rho) \Omega(n)$ \\
\hline CPOR-[6] & HomT & $\mathrm{O}(\mathrm{t})$ & $\mathrm{O}(\mathrm{t})$ & $\mathrm{O}(1)$ & & & $\#$ & $1-(1-\rho) t$ \\
\hline CPOR-II $\{6\}$ & HomT & $\mathrm{O}(\mathrm{t}+\mathrm{s})$ & $\mathrm{O}(\mathrm{t}+\mathrm{s})$ & $\mathrm{O}(\mathrm{s})$ & $\checkmark$ & & $\#$ & $1-(1-\rho) \mathrm{t}-\mathrm{s}$ \\
\hline OurScheme & Hom R & $\mathrm{O}(\mathrm{t}+\mathrm{c} . \mathrm{s})$ & $\mathrm{O}(\mathrm{t}+\mathrm{s})$ & $\mathrm{O}(\mathrm{s})$ & $\sqrt{ }$ & $\sqrt{ }$ & $\sqrt{ }$ & $\begin{array}{l}1-\Pi P k \in \mathcal{P} \\
(1-\rho k) r k \cdot t \cdot s\end{array}$ \\
\hline
\end{tabular}

$s$ is the number of sectors in each block, $c$ is the number of CSPs in a multi-cloud, t is the number of sampling blocks, $\rho$ and $\rho k$ are the probability of block corruption in a cloud server and $k$-th cloud server in a multi-cloud $\mathcal{P}=\{P k\}$, respective, \#denotes the verification process in a trivial approach, and MHT,HomT, HomR denotes Merkle Hash tree, homomorphic tags, and homomorphic response respectively.

some similar features: a single metadata server provides centralized management by a global namespace; files are split into blocks or chunks and stored on block servers; and the systems are comprised of interconnected clusters of block servers. Those features enable cloud service providers to store and process large amounts of data. However, it is crucial to offer an efficient verification on the integrity and availability of stored data for detecting faults and automatic recovery. Moreover, this verification is necessary to provide reliability by automatically maintaining multiple copies of data and automatically redeploying processing logic in the event of failures. Although existing techniques can make a false or true decision for data retention without downloading data at untrusted stores, they are not suitable for a distributed cloud storage environment since they were not originally constructed on interactive proof system. For example, the techniques based on Merkle Hash tree (MHT), such as Dynamic DDR-I, Dynamic DDR-II [1] and scalable DDR [4] in Table-1. Use an authenticated skip list to check the integrity of file blocks adjacently in space Unfortunately, they did not provide any algorithms for constructing distributed Merkle trees that are necessary for efficient verification in a multi-cloud environment. In addition, when a client asks for a file block, the server needs to send the file block along with a proof for the correctness of the block. However, this process incurs significant communication overhead in a multi-cloud environment, since the server in one cloud typically needs to generate such a proof with the help of other cloud storage services, where the adjacent blocks are stored. The other techniques, such as DDR [1], CPOR-I, and CPOR-II [6] in Table 1, are constructed on homomorphic verification tags, by which the server can generate tags for multiple file blocks in terms of a single response value. However, that doesn't mean the responses from multiple clouds can be also combined into a single value on the client side. In case of lack of homomorphic responses, clients must invoke the DDR protocol repeatedly to check the integrity of file blocks stored in multiple cloud servers. Also, clients need to know the exact position of each file block in a multi-cloud environment. In addition, the verification process in such a case will lead to high communication overheads and computation costs at client sides as well. Therefore, it is of utmost necessary to design a Cooperative DDR model to reduce the storage and network overheads and enhance the transparency of verification activities in cluster-based cloud storage systems. Moreover, such a Cooperative DDR technique should provide features for timely detecting abnormality and renewing multiple copies of data. Even though existing DDR techniques have addressed various security properties, such as public verifiability [1], dynamics [5], scalability [4], and privacy preservation [7], we still need a careful consideration of some potential attacks, including two major categories: Data Leakage Attack by which an adversary can easily obtain the stored data through verification process after running or wiretapping sufficient verification communications and Tag Forgery Attack by which a dishonest CSP can deceive the clients. These two attacks may cause potential risks for privacy leakage and ownership cheating. Also, these attacks can more easily compromise the security of a distributed cloud system than that of a single cloud system. Although various security models have been proposed for existing DDR techniques [1], [7], [6], these models still cannot cover all security requirements, especially for demonstrable secure privacy preservation and ownership verification. To establish a highly effective security model, it is necessary to analyze the DDR technique within the framework of zero-knowledge proof system (ZKPS) due to 
the reason that DDR system is essentially an interactive proof system (IPS), which has been well studied in the cryptography community. In summary, an verification technique for data integrity in distributed storage environments should have the following features: Usability aspect: A client should utilize the integrity check in the way of collaboration services. The technique should conceal the details of the storage to reduce the burden on clients; Security aspect: The technique should provide adequate security features to resist some existing attacks, such as data leakage attack and tag forgery attack; Performance aspect: The technique should have the lower communication and computation overheads than nonCooperative solution.

Related Works: To ensure the integrity and availability of outsourced data in cloud storages, researchers have proposed two basic approaches called Demonstrable data retention (DDR) [1] and Proofs of Retrievability (POR) [1]. Ateniese et al. [1] first proposed the DDR model for ensuring retention of files on untrusted storages and provided an RSA-based technique for a static case that achieves the (1) communication cost. They also proposed a publicly verifiable version, which allows anyone, not just the owner, to challenge the server for data retention. This property greatly extended application areas of DDR protocol due to the separation of data owners and the users. However, these techniques are insecure against replay attacks in dynamic scenarios because of the dependencies on the index of blocks. Moreover, they do not fit for multicloud storage due to the loss of homomorphism property in the verification process. In order to support dynamic data operations, Ateniese et al. developed a dynamic DDR solution called Scalable DDR [4]. They proposed a lightweight DDR technique based on cryptographic hash function and symmetric key encryption, but the servers can deceive the owners by using previous metadata or responses due to the lack of randomness in the challenges. The numbers of updates and challenges are limited and fixed in advance and users cannot perform block insertions anywhere. Based on this work, Erway etal. [5] Introduced two Dynamic DDR techniques with a hash function tree to realize $(\log n)$ communication and computational costs for a $n$-block file. The basic technique, called CDDR-I, retains the drawback of Scalable DDR, and in the 'blockless' technique, called CDDRII, the data blocks $\{m i j\} j \in[1, t]$ can be leaked by the response of a challenge, $M$ $=\sum_{j=1}^{t} a j m i j$, where $a j$ is a random challenge value. Furthermore, these techniques are also not effective for a multi-cloud environment because the verification path of the challenge block cannot be stored completely in a cloud [8]. Juels and Kaliski [3] presented a POR technique, which relies largely on preprocessing steps that the client conducts before sending a file to a CSP. Unfortunately, these operations prevent any efficient extension for updating data. Shacham and Waters [6] proposed an improved version of this protocol called Compact POR, which uses homomorphic property to aggregate a proof into (1) authenticator value and $O(t)$ computation cost for $t$ challenge blocks, but their solution is also static and could not prevent the leakage of data blocks in the verification process. Wang et al. [7] presented a dynamic technique with $(\log n)$ cost by integrating the Compact POR technique and Merkle Hash Tree (MHT) into the CDDR. Furthermore, several POR techniques and models have been recently proposed including [9], [10]. In [9] Bowers et al. introduced a distributed cryptographic system that allows a set of servers to solve the DDR problem. This system is based on an integrity-protected error Correcting code (IP-ECC), which improves the security and efficiency of existing tools, like POR. However, a file must be transformed into $l$ distinct segments with the same length, which are distributed across $l$ servers. Hence, this system is more suitable for RAID rather than cloud storage. Our Contributions, in this paper, we address the problem of demonstrable data retention in distributed cloud environments from the following aspects: high performance, transparent verification, and high security. To achieve these goals, we first propose a verification framework for multi-cloud storage along with two fundamental techniques: homomorphic verifiable response (HVR) and hash index hierarchy (HIH). We then demonstrate that the possibility of constructing a Cooperative DDR (CDDR) technique without compromising data privacy based on modern cryptographic techniques, such as interactive proof system (IPS). We further introduce an effective construction of CDDR technique using above-mentioned structure. Moreover, we give a security analysis of our CDDR technique from the IPS model. We prove that this construction is a multiprover zero-knowledge proof system (MP-ZKPS) [11], which has zero-knowledge properties, completeness and knowledge soundness. These properties ensure that CDDR technique can implement the security against data leakage attack and tag forgery attack. To improve the system performance with respect to our technique, we analyze the performance of probabilistic queries fordetecting abnormal situations. This probabilistic method also has an inherent benefit in reducing computation and communication overheads. Then, we present an efficient method for the selection of optimal parameter values to minimize the computation overheads of CSPs and the clients' operations. Inaddition, we analyze that our technique is suitable for existing distributed cloud storage systems. Finally, our experiments show that our solution introduces very limited computation and communication overheads. 
Organization: The rest of this paper is organized as follows. In Section 2, we describe a formal definition of CDDR and the underlying techniques, which are utilized in the construction of our technique. We introduce the details of Cooperative DDR technique for multicloud storage in Section 3. We describe the security and performance evaluation of our technique in Section 4 and 5 , respectively. We discuss the related work in Section and Section 6 concludes this paper.

\section{STRUCTURE AND TECHNIQUES}

In this section, we present our verification framework for multi-cloud storage and a formal definition of CDDR. We introduce two fundamental techniques for constructing our CDDR technique: hash index hierarchy $(\mathrm{HIH})$ on which the responses of the clients' challenges computed from multiple CSPs can be combined into a single response as the final result; and homomorphic verifiable response (HVR) which supports distributed cloud storage in a multicloud storage and implements an efficient construction of collision resistant hash function, which can be viewed as a random oracle model in the verification protocol.

Fig 1: Verification architecture for data integrity.

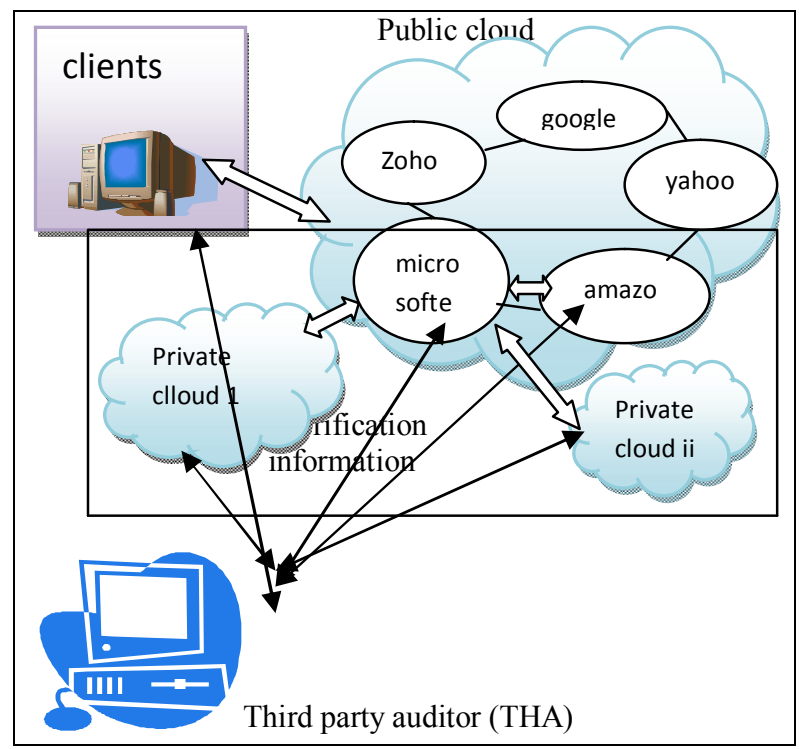

\subsection{Verification Framework for Multi-}

Cloud:Although existing DDR techniques offer a publicly accessible remote interface for checking and managing the tremendous amount of data, the majority of existing DDR techniques is incapable to satisfy the inherent requirements from multiple clouds in terms of communication and computation costs. To address this problem, we consider amulti-cloud storage service as illustrated in Figure 1. In this architecture, a data storage service involves three different entities: Clients who have a large amount of data to be stored in multiple clouds and have the permissions to access and manipulate stored data; Cloud Service Providers (CSPs) who work together to provide data storage services and have enough storages and computation resources; and Trusted Third Party (TTP) who is trusted to store verification parameters and offer public query services for these parameters. In this architecture, we consider the existence of multiple CSPs to Cooperative store and maintain the clients' data. Moreover, a Cooperative DDR is used to verify the integrity and availability of their stored data in all CSPs. The verificationprocedure is described as follows: Firstly, a client (data owner) uses the secret key to pre-process a file which consists of a collection of $n$ blocks, generates a set of public verification information that is stored in TTP, transmits the file and some verification tagsto CSPs, and may delete its local copy; Then, by using a verification protocol, the clients can issue a challenge for one CSP to check the integrity and availability of outsourced data with respect to public information stored in TTP. We neither assume that CSP is trust to guarantee the security of the stored data, nor assume that data owner has the ability to collect the evidence of the CSP's fault after errors have been found. To achieve this goal, a TTP server is constructed as a core trust base on the cloud for the sake of security We assume the TTP is reliable and independent through the following functions [12]: to setup and maintain the CDDR cryptosystem; to generate and store data owner's public key; and to store the public parameters used to execute the verification protocol in the CDDR technique. Note that the TTP is not directly involved in the CDDR technique in order to reduce the complexity of cryptosystem.

2.2 Definition of Cooperative DDR: In order to prove the integrity of data stored in a multi-cloud environment, we define a framework for CDDR based on interactive proof system (IPS) and multi-prover zeroknowledge proof system (MPZKPS), as follows:Definition 1 (Cooperative-DDR): A Cooperative demonstrable data retention $\mathcal{S}=($ KeyGen, TagGen, Proof $)$ is a collection of twoalgorithms (KeyGen,TagGen) and an interactive proof system Proof, as follows: $\left(1^{k}\right)$ : takes a security parameter $k$ as input, and returns a secret key $s k$ or a public-secret key-pair ( $p k, s k)$; TagGen $(s k, F, \mathcal{P})$ : takes as inputs a secret key $s k$, a file $F$, and a set of cloud storage providers $\mathcal{P}=\{P k\}$, and returns the triples $(\zeta, \psi, \sigma)$, where $\zeta$ isthe secret in tags, $\psi=(u, \mathcal{H})$ is a set of verification parameters $u$ and an index hierarchy $\mathcal{H}$ for $F, \sigma=$ $\left\{\sigma^{(k)}\right\} p_{k} \in \mathcal{P}$ denotes a set of all tags, $\sigma^{(k)}$ is the tag of the fraction $F^{(k)}$ of $F$ in $P_{k} ;(\mathcal{P}, \mathbf{V})$ : is a protocol of proof of 
data retention between CSPs $\left(\mathcal{P}=\left\{P_{k}\right\}\right)$ and a verifier (V), that is, $\quad\left\langle\quad \sum_{P_{k} \in p} P_{k}\left(F^{(k)}, \sigma^{(k)} \quad\right) \leftrightarrow V\right\rangle(p k, \psi)$ $=\left\{\begin{array}{l}1, F=\left\{F^{(k)}\right\} \text { isintact } \\ 0, F=\left\{F^{(k)}\right\} \text { ischanged }\end{array}\right.$ Where each $P_{k}$ takes as input a file $F^{(k)}$ and a set of tags $\sigma^{(k)}$, and a public key $p k$ and a set of public parameters $\psi$ are the common input between $P$ and $V$. At the end of the protocol run, $V$ returns a bit $\{1 \mid 0\}$ denoting true and false. Where, $\sum P_{k} \in p$ denotes Cooperative computing in $P_{k} \in \mathcal{P}$. A trivial way to realize the CDDR is to check the data stored in each cloud one by one, i.e. $\wedge_{P_{k} \in p}\left\langle P_{k}\left(F^{(k)}, \sigma^{(k)} \leftrightarrow \mathrm{V}\right\rangle(\mathrm{pk}, \psi)\right.$ Where $\Lambda$ denotes the logical AND operations among the Boolean outputs of all protocols $\left\langle P_{k}, V\right\rangle$ for all $P_{k} \in \mathcal{P}$. However, it would cause significant communication and computation overheads for the verifier, as well as a loss of location-transparent. Such a primitive approach obviously diminishes the advantages of cloud storage: scaling arbitrarily up and down on demand [13]. To solve this problem, we extend above definition by adding an organizer $(O)$, which is one of CSPs that directly contacts with the verifier, as follows: $\left\langle\sum_{P_{k} \in p} P_{k}\left(F^{(k)}, \sigma^{(k)}\right)\right.$ $\leftrightarrow \mathrm{O} \leftrightarrow V\rangle(p k, \psi)$, Where the action of organizer is to initiate and organize the verification process. This definition is consistent with aforementioned architecture, e.g., a client (or an authorized application) is considered as, the CSPs are as $\mathcal{P}=\left\{P_{i}\right\} i \in[1, c]$, and the Zoho cloud is as the organizer in Figure 1. Often, the organizer is an independent server or a certain CSP in $\mathcal{P}$. The advantage of this new multi-prover proof system is that it does not make any difference for the clients between multi-prover verification process and single-prover verification process in the way of collaboration. Also, this kind of transparent verification is able to conceal the details of data storage to reduce the burden on clients. For the sake of clarity, we list some used signals in Table 2.

TABLE 2: The signal and its explanation.

\begin{tabular}{|l|l|}
\hline Sig. & Repression \\
\hline $\boldsymbol{n}$ & the number of blocks in a file; \\
\hline $\boldsymbol{s}$ & the number of sectors in each block; \\
\hline $\boldsymbol{t}$ & the number of index coefficient pairs in a query; \\
\hline $\boldsymbol{c}$ & the number of clouds to store a file; \\
\hline $\boldsymbol{F}$ & $\begin{array}{l}\text { the file with } n \times s \text { sectors, i.e., } F=\{\mathrm{mi}, j\} i \in[1, n] \\
, j \in[1, s] ;\end{array}$ \\
\hline $\boldsymbol{\sigma}$ & the set of tags, i.e., $\sigma=\{\sigma i\} i \in[1, n] ;$ \\
\hline $\boldsymbol{Q}$ & the set of index-coefficient pairs, i.e., $Q=\{(i, v i)\} ;$ \\
\hline $\boldsymbol{\theta}$ & the response for the challenge $Q$. \\
\hline
\end{tabular}

2.3 Hash Index Hierarchy for CDDR:To support distributed cloud storage, we illustrate a representative architecture used in our Cooperative DDR technique as shown in Figure 2. Our architecture has a hierarchy structure which resembles a natural representation of file storage. This hierarchical structure $\mathcal{H}$ consists of three layers to represent relationships among all blocks for stored resources. They are described as follows: 1) Express Layer: offers an abstract representation of the stored resources; 2) Service Layer: offers and manages cloud storage services; and 3) Storage Layer: realizes data storage on many physical devices. We make use of this simple hierarchy to organize data blocks from multiple CSP services into a large size file by shading their differences among these cloud storage systems. For example, in Figure 2 the resources in Express Layer are split and stored into three CSPs, which are indicated by different colors, in Service Layer. In turn, each CSP fragments and stores the assigned data into the storage servers in Storage Layer. We also make use of colors to distinguish different CSPs. Moreover, we follow the logical order of the data blocks to organize the Storage Layer. This architecture also provides special functions for data storage and management, e.g., there may exist overlaps among data blocks (as shown in dashed boxes) and discontinuous blocks but these functions may increase the complexity of storage management. In storage layer, we define a common fragment structure that provides probabilistic verification of data integrity for outsourced storage. The fragment structure is a data structure that maintains a set of block-tag pairs, allowing searches, checks and updates in (1) time. An instance of this structure is shown in storage layer of Figure 2: an outsourced file $F$ is split into $n$ blocks $\{m 1, \mathrm{~m} 2, \cdots$,$\} , and$ each block $m i$ is split into $s$ sectors $\{m i, 1, m i, 2, \cdots, m i, s\}$. The fragment structure consists of $n$ block-tag pair $\left(m_{i}, \sigma_{i}\right)$, where $\sigma_{i}$ is a signature tag of block $m_{i}$ generated by a set of secrets $\tau=\left(\tau_{1}, \tau_{2}, \cdots, \tau_{s}\right)$. In order to check the data integrity, the fragment structure implements probabilistic verification as follows: given a random chosen challenge (or query) $Q=\left\{\left(i, v_{i}\right)\right\} i \in R I$, where $I$ is a subset of the block indices and $v_{i}$ is a random coefficient. There exists an efficient algorithm to produce a constant-size response $\left(\mu_{1}, \mu_{2}, \cdots, \mu_{s}, \sigma^{\prime}\right)$, where $\mu_{i}$ comes from all $\left\{m_{k}, i, v_{k}\right\} \mathrm{k} \in \mathrm{I}$ and $\sigma^{\prime}$ is from all $\left\{\sigma_{k}, v_{k}\right\} \mathrm{k} \in \mathrm{I}$. Given a collision-resistant hash function $H_{k}(\cdot)$, we make use of this architecture to construct a Hash Index Hierarchy $\mathcal{H}$ (viewed as a random oracle), which is used to replace the common hash function in prior DDR techniques, as follows:1) Express layer: given $s$ random $\left\{\tau_{i}\right\}_{i=1}^{s}$ and the file name $F_{n}$, sets $\xi^{(1)}=$ $H_{\sum_{i}^{s} \tau_{i}} F_{n}=1$ and makes it public for verification but makes $\left\{\tau_{i}\right\}_{i=1}^{s}$ secret;2) Service layer: given the $\xi^{(1)}$ and the cloud name $C_{k}$, sets $\left.\xi^{(2)}=H_{\xi^{(1)}}\left(C_{k}\right) ; 3\right)$ Storage layer: given the $\xi^{(2)}$, a blocknumber $\mathrm{i}$, and its index record $X_{i}=$ " $B_{i}\left\|V_{i}\right\| R_{i}$ ", sets $\xi_{i, k}^{(3)}=H_{\xi_{i, k}^{(2)}}\left(X_{i}\right)$, where $B_{i}$ is the sequence number of a block, $V_{i}$ is the updated version number, and $R_{i}$ is a random integer to avoid collision. As a virtualization approach, we introduce a simple index-hash table $\mathrm{X}=\left\{X_{i}\right\}$ 
to record the changes of file blocks as well as to generate the hash value of each block in the verification process. The structure of $\mathrm{X}$ is similar to the structure of file block allocation table in file systems. The index-hash table consists of serial number, block number, version number, random integer, and so on. Different from the common index table, we assure that all records in our index table to differ from one another prevent forgery of data blocks and tags. By using this structure, especially the index records

Fig 2:Index-hash hierarchy of CDDR model.
$\left\{X_{i}\right\}$, our CDDR technique can also support dynamic data operations [8].The proposed structure can be readily incorporated into MAC-based, ECC or RSA techniques [1], [6]. These techniques, built from collision-resistance signatures (see Section 3.1) and the random oracle model, have the shortest query and response with publicverifiability. They share several common characters for theimplementation of the CDDR framework in the multiple clouds: 1) a file is split into $n \times s$ sectors

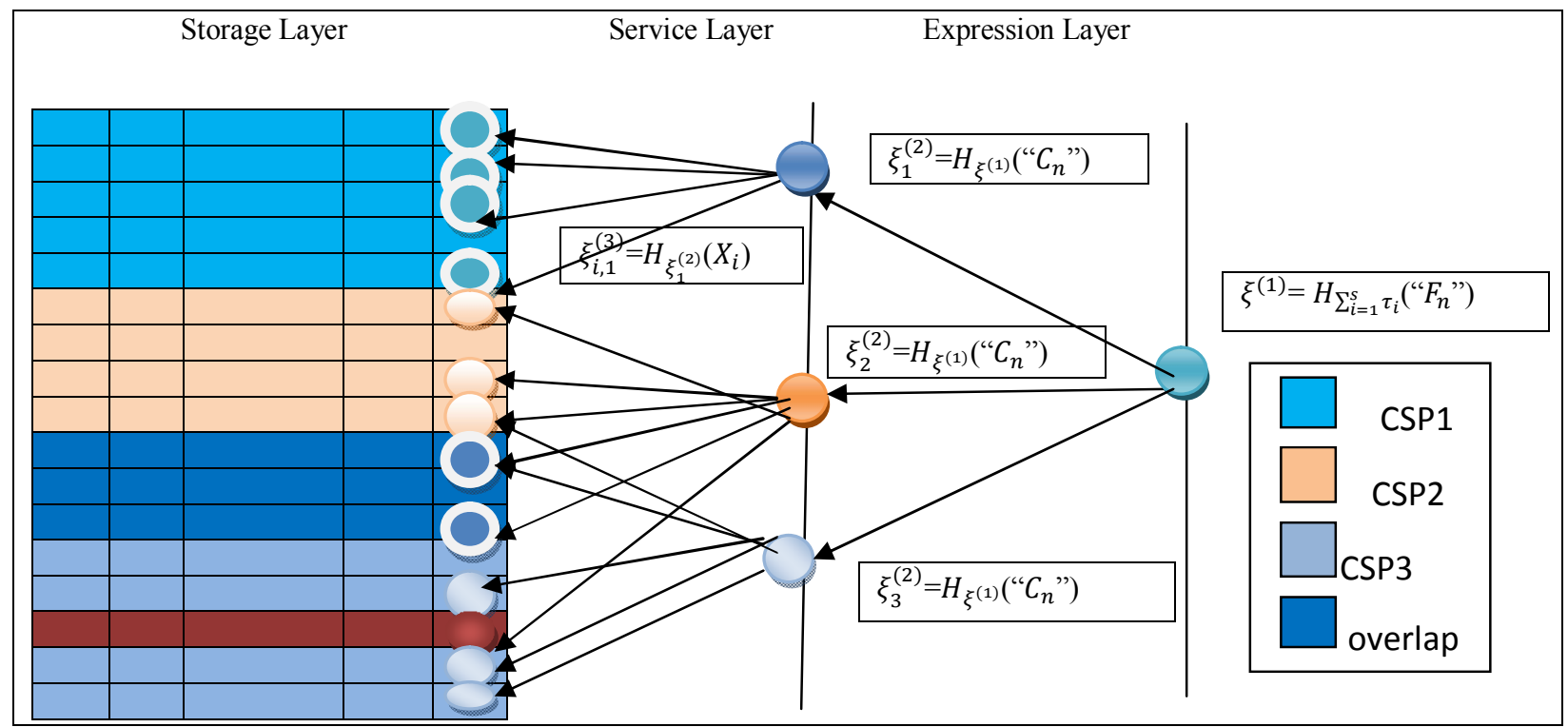

and each block ( $s$ sectors) corresponds to a tag, so that the storage of signature tags can be reduced by the increase of $s ; 2)$ a verifier can verify the integrity of file in random sampling approach, which is of utmost importance for large files; 3) these techniques rely on homomorphic properties to aggregate data and tags into a constant size response, which minimizes the overhead of network communication; and 4) the hierarchy structure provides a virtualization approach to conceal the storage details of multiple CSPs.

\subsection{Homomorphic Verifiable Response for}

CDDR:A homomorphism is a map $f: \mathbb{P} \rightarrow \mathbb{Q}$ between two groups such that $f\left(g_{1 \oplus} g_{2}\right)=f\left(g_{1}\right) \otimes f\left(g_{2}\right)$ for all $g_{1}$, $g_{2} \in \mathbb{P}$, where $\oplus$ denotes the operation in $\mathrm{P}$ and $\otimes$ denotes the operation in $\mathbb{Q}$. This notation has been used to define Homomorphic Verifiable Tags (HVTs) in [1]: Given two values $\sigma_{i}$ and $\sigma_{j}$ for two messages $m_{i}$ and $m_{j}$, anyone can combine them into a value $\sigma_{i}{ }^{\prime}$ corresponding to the sum of the messages $m_{i}+m_{j}$. When demonstrable data retention is considered as a challenge-response protocol, we extend this notation to the concept of Homomorphic Verifiable
Responses (HVR), which is used to integrate multiple responses from the different CSPs in CDDR

technique as follows: Definition 2 (HVR): A response is called homomorphic verifiable response in a DDR protocol, if given two responses $\Theta_{i}$ and $\Theta_{j}$ for two challenges $Q_{i}$ and $Q_{j}$ from two CSPs, there exists an efficient algorithm to combine them into a response $\Theta$ corresponding to the sum of the challenges $Q_{i} \cup Q_{j}$. Homomorphic verifiable response is the key technique of CDDR because it not only reduces the communication bandwidth, but alsoconceals the location of outsourced data in the distributed cloud storage environment.

\section{COOPERATIVE DDR TECHNIQUES}

In this section, we propose a CDDR technique for multicloud system based on the above-mentioned structure and techniques. This technique is constructed on collisionresistant hash, bilinear map group, aggregation algorithm, and homomorphic responses. 
3.1 Notations and Preliminaries: Let $\mathbb{H}=\left\{H_{k}\right\}$ be a family of hash functions $H_{k}:\{0,1\}^{n} \rightarrow\{0,1\}^{*}$ index by $k \in \mathcal{K}$. We say that algorithm $\mathcal{A}$ has advantage $\epsilon$ in breaking collision resistance of $\mathbb{H}$ if $\operatorname{Pr}\left[\mathcal{A}(k)=\left(m_{0}, m_{1}\right): m_{0}=\right.$ $\left.m_{1}, H_{k}\left(m_{0}\right)=H_{k}\left(m_{0}\right)\right] \geq \epsilon$, where the probability is over the random choices of $k \in \mathcal{K}$ and the random bits of $\mathcal{A}$. So that, we have the following definition: Definition 3 (Collision-Resistant Hash): A hash family $\mathbb{H}$ is $(t, \epsilon)$ collision-resistant if no $t$-time adversary has advantage at least $\epsilon$ in breaking collision resistance of $\mathbb{H}$. We set up our system using bilinear pairings proposed by Boneh and Franklin [12]. Let $\mathbb{G}$ and $\mathbb{G} T$ be two multiplicative groups using elliptic curve conventions with a large prime order $p$. The function $e$ is a computable bilinear map $e: \mathbb{G} \times \mathbb{G} \rightarrow$ $\mathbb{G} T$ with the following properties: for any $G, H \in \mathbb{G}$ and all $a$, $b \in \mathbb{Z} p$, we have 1) Bilinearity: $e([a] G,[b] H)=e(G, H) a b$; 2) Non-degeneracy: $e(G, H) \neq 1$ unless $G$ or $H=1$; and 3) Computability: $e(G, H)$ is efficiently computable.Definition 4 (Bilinear Map Group System): A bilinear map group system is a tuple $\mathbb{S}=\langle p,,, e\rangle$ composed of the objects as described above.

3.2 Our CDDR Technique:In our technique (see Fig 3), the manager first runs algorithm KeyGen to obtain the public/private key pairs for CSPs and users. Then, the clients generate the tags of outsourced data by using TagGen. Anytime, the protocol Proof is performed by a 5-move interactive Proof protocol between a verifier and more than one CSP, in which CSPs need not to interact with each other during the verification process, but an organizer, is used to organize and manage all CSPs. This protocol can be described as follows: 1) The organizer initiates the protocol and sends a commitment to the verifier; 2) The verifier returns a challenge set of random index-coefficient pair's $Q$ to the organizer; 3) The organizer relays them into each lock; 4) Each $P i$ returns its response of challenge to the organizer; and 5) The organizer synthesizes aP $i$ in $\mathcal{P}$ according to the exact position of each data final response from received responses and sends it to theverifier. The above process would guarantee that the verifier accesses files without knowing on which CSPs or in what geographical locations their files reside. In contrast to a single CSP environment, our technique differs from the common DDR technique in two aspects:1) Tag aggregation algorithm: In stage of commitment, the organizer generates a random $\gamma \in R \mathbb{Z} p$ and returns its commitment $\mathrm{H}^{\prime}{ }_{1}$ to the

\subsection{Collision resistant for index-hash}

hierarchy:In our CDDR technique, the collision resistant of index hash hierarchy is the basis and prerequisite for the security of whole technique, which is described as being secure in the random oracle model. Although the hash function is collision resistant, a verifier. This assures that the verifier and CSPs do not obtain the value of $\gamma$. Therefore, our approach guarantees only the organizer can compute the final $\sigma^{\prime}$ by using $\gamma$ and $\sigma^{\prime} k$ received from CSPs. After $\sigma^{\prime}$ is computed, we need to transfer it to the organizer in stage of "Response1". In order to ensure the security of transmission of data tags, our technique employs a new method, similar to the ElGamal encryption, to encrypt the combination of tags $\prod_{\left(i, v_{i}\right) \in Q_{k}} \sigma_{i}^{v_{i}}$, that is, for $s k=s \in \mathbb{Z} p$ and $P_{k}=\left(g, S=g^{s}\right)$ $\in \mathbb{G}^{2}$, the cipher of message $m$ is $\mathcal{C}=\left(\mathcal{C}_{1}=g r, \mathcal{C}_{2}=m \cdot s^{r}\right)$ and its decryption is performed by $\left.m=C_{2} . C_{1}^{-s} .2\right)$ Homomorphic responses: Because of the homomorphic property, the responses computed from CSPs in a multicloud can be combined into a single final response. It is obvious that the final response $\theta$ received by the verifiers from multiple CSPs is same as that in one simple CSP. This means that our CDDR technique is able to provide a transparent verification for the verifiers. Two response algorithms, Response1 and Response2, comprise an HVR: Given two responses $\theta i$ and $\theta j$ for two challenges $Q i$ and $Q j$ from two CSPs, i.e., $\theta i=$ Response 1 ( $Q i,\{m k\} k \in I i$, $\{\sigma k\} k \in I i)$, there exists an efficient algorithm to combine them into a final response $\theta$ corresponding to the sum of the challenges $Q i \cup$, that is, = Response $1(Q i \cup,\{m k\}$ $k \in I i \cup I j,\{\sigma k\} k \in I i \cup I j)=$ Response $2\left(\theta_{i}, \theta_{j}\right)$. For multiple CSPs, the above equation can be extended to $\theta=$ Response2 $(\{\theta k\} \in \mathcal{P})$. More importantly, the HVR is a pair of values $\theta=(\pi, \sigma, \mu)$, which has a constant-size even for different challenges.

4 SECURITY ANALYSESWe give a brief security analysis of our CDDR construction. This construction is directly derived from multi-prover zeroknowledge proof system (MPZKPS), which satisfies following properties for a given assertion, $L$ : 1) Completeness: whenever $x \in L$, there exists a strategy for the provers that convinces the verifier that this is the case;

2) Soundness: whenever $x \notin L$, whatever strategy the provers employ, they will not convince the verifier that $x \in L ; 3)$ Zero-knowledge: no cheating verifier can learn anything other than the veracity of the statement. According to existing IPS research [11], these properties can protect our construction from various attacks, such as data leakage attack (privacy leakage), tag forgery attack (ownership cheating), etc. In details, the security of our technique can be analyzed as follow successful hash collision can still be used to produce a forged tag when the same hash value is reused multiple times, e.g., a legitimate client modifies the data or repeats to insert and delete data blocks of outsourced data. To avoid the hash collision, the hash value $\xi(3) i, k$, which is used to generate the tag $\sigma i$ in CDDR technique, is computed from the set of values $\{\tau i\}, F n, C k,\{\chi i\}$. As 
long as there exists one bit difference in these data, we can avoid the hash collision. As a consequence, we have the following theorem (see Appendix B): Theorem 1 (Collision Resistant): The index-hash hierarchy in CDDR technique is collision resistant, even if the client generates $\sqrt{2 p \cdot \ln \frac{1}{1-\varepsilon}}$ files with the same file name and cloud name, and the client repeats $\sqrt{2^{L+1} \cdot \ln \frac{1}{1-\varepsilon}}$ times to modify, insert and delete data blocks, where the collision probability is at least $\varepsilon, \tau i \in \mathbb{Z} p$, and $|R i|=L$ for $R i \in \chi i$.

\subsection{Completeness property of verification:In} our technique, the completeness property implies public verifiability property, which allows anyone, not just the client (data owner), to challenge the cloud server for data integrity and data ownership without the need for any secret information. First, for every available data-tag pair $(F, \sigma) \in(s k, F)$ and a random challenge $Q=(\mathrm{i}, v i) i \in I$, the verification protocol should be completed with success probability according to the Equation (3), that is, $\operatorname{Pr}$ $\left[\left\langle\sum_{P_{k} \in p} P_{k}\left(F^{(k)}, \sigma^{(k)}\right) \leftrightarrow 0 \leftrightarrow \mathrm{V}\right\rangle(\mathrm{pk}, \psi)=1\right]=1$. In this process, anyone can obtain the owner's public key $p k=(g$, $\left.h, H_{1}=h^{\alpha}, H_{2}=h^{\beta}\right)$ and the corresponding file parameter $\psi$ $=\left(u, \xi^{(1)}, \chi\right)$ from TTP to execute the verification protocol, hence this is a public verifiable protocol. Moreover, for different owners, the secrets $\alpha$ and $\beta$ hidden in their public key $p k$ are also different, determining that success verification can only be implemented by the real owner's public key. In addition, the parameter $\psi$ is used to store the file-related information, so an owner can employ a unique public key to deal with a large number of outsourced files.

\subsection{Zero-knowledge property of verification:}

The CDDR construction is in essence a Multi-Prover Zeroknowledge Proof (MP-ZKP) system [11], which can be considered as an extension of the notion of an interactive proof system (IPS). Roughly speaking, in the scenario of MP-ZKP, a polynomial-time bounded verifier interacts with several provers whose computational powers are unlimited. According to a Simulator model, in which every cheating verifier has a simulator that can produce a transcript that "looks like" an interaction between an honest prover and a cheating verifier, we can prove our CDDR construction has Zero-knowledge property.

Theorem 2 (Zero-Knowledge Property): The verification protocol $\operatorname{Proof}(\mathcal{P}, V)$ in $\operatorname{CDDR}$ technique is a computational zero-knowledge system under a simulator model, that is, for every probabilistic polynomial-time interactive machine $V *$, there exists a probabilistic polynomial-time algorithm $S *$ such that the ensembles View $(\langle\Sigma P k \in \mathcal{P P} k(F(k), \sigma(k)) \leftrightarrow O \leftrightarrow V *\rangle(p k, \psi))$ and
$S *(p k, \psi)$ are computationally indistinguishable. Zeroknowledge is a property that achieves the CSPs' robustness against attempts to gain knowledge by interacting with them. For our construction, we make use of the zeroknowledge property to preserve the privacy of data blocks and signature tags. Firstly, randomness is adopted into the CSPs' responses in order to resist the data leakage attacks (see Attacks 1 and 3 in Appendix A). That is, the random integer $\lambda j$, is introduced into the response $\mu j$, i.e., $\mu j, k=$ $\lambda j, k+\Sigma(i, v i) \in Q k v i \cdot m i, j$. This means that the cheating verifier cannot obtain $m i$, from $\mu j$, because he does not know the random integer $\lambda j$. At the same time, a random integer $\gamma$ is also introduced to randomize the verification $\operatorname{tag} \sigma$, i.e., $\sigma^{\prime} \leftarrow\left(\Pi \mathrm{Pk} \in \mathcal{P} \sigma^{\prime} \mathrm{k} \cdot \mathrm{R}-\mathrm{s} \mathrm{k}\right)^{\gamma}$. Thus, the tag $\sigma$ cannot reveal to the cheating verifier in terms of randomness.

\subsection{Knowledge soundness of verification:For} every data-tag pairs $(F *, \sigma *) / \in(s k, F)$, in order to prove nonexistence of fraudulent $\mathcal{P} *$ and $O *$, we require that the technique satisfies the knowledge soundness property, that is, $\operatorname{Pr}\left[\left\langle\sum_{P_{k} \in p^{*}} P_{k}\left(F^{(k) *}, \sigma^{(k) *}\right) \leftrightarrow 0^{*} \leftrightarrow \mathrm{V}\right\rangle(\mathrm{pk}, \psi)=1\right] \leq$ $\epsilon$, where $\epsilon$ is a negligible error. We prove that our technique has the knowledge soundness property by using reduction to absurdity 1 : we make use of $\mathcal{P} *$ to construct a knowledge extractor $\mathcal{M}[7,13]$, which gets the common input $(p k, \psi)$ and rewindable blackbox accesses to the prover $P *$, and then attempts to break the computational Diffie-Hellman (CDH) problem in $\mathbb{G}$ : given $G, G_{1}=G^{a}, G_{2}$ $=G^{b} \in R \mathbb{G}$, output $G a b \in \mathbb{G}$. But it is unacceptable because the problem in polynomial-time.

Theorem 3 (Knowledge Soundness Property): Our technique has $\left(t, \epsilon^{\prime}\right)$ knowledge soundness in random oracle and rewindable knowledge extractor model assuming the $(t, \epsilon)$-computational Diffie-Hellman $(\mathrm{CDH})$ assumption holds in the group $\mathbb{G}$ for $\epsilon^{\prime} \geq \epsilon$. Essentially, the soundness means that it is infeasible to fool the verifier to accept false statements. Often, the soundness can also be regarded as a stricter notion of unforgeability for file tags to avoid cheating the ownership. This means that the CSPs, even if collusion is attempted, cannot be tampered with the data or forge the data tags if the soundness property holds. Thus, the Theorem 3 denotes that the CDDR technique can resist the tag forgery attacks.

\section{PERFORMANCE EVALUATIONS}

In this section, to detect abnormality in a low overhead and timely manner, we analyze and optimize the performance of CDDR technique based on the above technique from two aspects: evaluation of probabilistic queries and optimization of length of blocks. To validate the effects of 
technique, we introduce a prototype of CDDR-based audit system and present the experimental results.

\subsection{Performance Analysis for CDDR}

Technique:We present the computation cost of our CDDR technique in Table 3. We use $[E]$ to denote the computation cost of an exponent operation in $\mathbb{G}$, namely, $g x$, where $x$ is a positive integer in $\mathbb{Z} p$ and $g \in \mathbb{G}$ or $\mathbb{G} T$. We neglect the computation cost of algebraic operations and simple modular arithmetic operations because they run fast enough [12]. The most complex operation is the computation of a bilinear map $(\cdot, \cdot)$ between two elliptic points (denoted as $[B]$ ). Then, we analyze the storage and communication costs of our technique. We define the bilinear pairing takes the form: $(\mathbb{F p m}) \times(\mathbb{F p k m}) \rightarrow$ $\mathbb{F} * p k m$ (The definition given here is from [13], [8]), where $p$ is a prime, $m$ is a positive integer, and $k$ is the embedding degree (or security multiplier). In this case, we utilize an asymmetric pairing: $\mathbb{G} 1 \times \mathbb{G} 2 \rightarrow \mathbb{G} T$ to replace the symmetric pairing in the original techniques. In Table 3 , it is easy to find that client's computation overheads are entirely irrelevant for the number of CSPs. Further, our technique has better performance compared with non-Cooperative numberof CSPs.Further, our technique has better

TABLE 3: Comparison of computation overheads between our CDDR scheme and non-cooperative (trivial) scheme.

\begin{tabular}{|l|l|l|}
\hline & \multicolumn{1}{|r|}{ CDDR Scheme } & Trivial Scheme \\
\hline Commitment & $l 2$ & $c l 2$ \\
\hline Challenge 1 & $2 t l 0$ & $2 t l 0$ \\
\hline Challenge2 & $2 t l 0 / c$ & $2 t l 0$ \\
\hline Response1 & $s l 0+2 l 1+l T$ & $(s l 0+l 1+l T) c$ \\
\hline Response2 & $s l 0+l 1+l T$ & $(s l 0+l 1+l T) c$ \\
\hline
\end{tabular}

performance compared with non-Cooperative approach due to the total of computation overheads decrease $3(c-1)$ times bilinear map operations, where $c$ is the number of clouds in a multi-cloud. The reason is that, before the responses are sent to the verifier from $c$ clouds, the organizer has aggregate these responses into a response by using aggregation algorithm, so the verifier only need to verify this response once to obtain the final result. Without loss of generality, let the security parameter $\kappa$ be 80 bits, we need the elliptic curve domain parameters over $\mathbb{F} p$ with $|p|=$ 160 bits and $m=1$ in our experiments. This means that the length of integer is $l 0=2 \kappa$ in $\mathbb{Z} p$. Similarly, we have $l 1=$ $4 \kappa$ in $\mathbb{G} 1, l 2=24 \kappa$ in $\mathbb{G} 2$, and $l T=24 \kappa$ in $\mathbb{G T}$ for the embedding degree $k=6$. The storage and communication cost of our technique is shown in Table 4. The storage overhead of a file with $(f)=1 M$-bytes is $(f)=n \cdot s \cdot l 0+n \cdot l 1$ $=1.04 M$-bytes for $n=103$ and $s=50$. The storage overhead of its index table $\chi$ is $n \cdot l 0=20 K$-bytes. We define the overhead rate as $\lambda=(f)(f)-1=l 1 s \cdot l 0$ and it should therefore be kept as low as possible in order to minimize the storage in cloud storage providers. It is obvious that a higher $s$ means much lower storage. Furthermore, in the verification protocol, the communication overhead of challenge is $2 t \cdot l 0=40 \cdot t$-Bytes in terms of the number of challenged blocks $t$, but its response (response1 or response2) has a constant-size communication overhead $s \cdot l 0+l 1+l T \approx 1.3 \mathrm{~K}$-bytes for different file sizes. Also, it implies that client's communication overheads are of a fixed size, which is entirely irrelevant for the number of CSPs.

TABLE 4: Comparison of communication overheads between our CDDR and non-cooperative scheme

\begin{tabular}{|l|l|l|}
\hline & CDDR Scheme & Trivial Scheme \\
\hline KeyGen & $3[E]$ & $2[E]$ \\
\hline TagGen & $(2 n+s)[E]$ & $(2 n+s)[E]$ \\
\hline Proof $(\mathrm{p})$ & $c[B]+(t+c s+1)[E]$ & $c[B]+(t+c s-c)[E]$ \\
\hline Proof(V) & $3[B]+(t+s)[E]$ & $3 c[B]+(t+c s)[E]$ \\
\hline
\end{tabular}

5.2 Probabilistic Verification:We recall the probabilistic verification of common DDR technique (which only involves one CSP), in which the verification process achieves the detection of CSP server misbehavior in a random sampling mode in order to reduce the workload on the server. The detection probability of disrupted blocks $P$ is an important parameter to guarantee that these blocks can be detected in time. Assume the CSP modifies eblocks out of the $n$-block file, that is, the probabilityof disruptedblocks is $\rho b=e n$. Let $t$ be the number of queriedblocks for a challenge in the verification protocol. We have detection probability ${ }^{2}(\rho b, t) \geq 1-\left(\frac{n-e}{n}\right)^{t}=$ $1-(1-\rho b)^{t}$, Where, $(\rho b, t)$ denotes that the probability $P$ is a function over $\rho b$ and $t$. Hence, the number of queried blocks is $t \approx \frac{\log (1-\mathrm{P})}{\log (1-\rho \mathrm{b})} \approx \frac{\mathrm{P} \cdot \mathrm{n}}{\mathrm{e}}$ for a sufficiently large $n$ and $t \ll \mathrm{n}^{3}$. This means that the number of queried blocks $t$ is directly proportional to the total number of file blocks $n$ for the constant $P$ and $e$. Therefore, for a uniform random verification in aDDR technique with fragment structure, given a file with $S Z=n \cdot s$ sectors and the probability of sector corruption $\rho$, the detection probability of verification protocol has $P \geq 1-(1-\rho)^{\mathrm{SZ} \cdot \omega}$, where $\omega$ denotes the sampling probability in the verification protocol. We can obtain this result as follows: because $\rho b \geq 1-(1-\rho)^{\mathrm{s}}$ is the probability of block corruption with $s$ sectors in common DDR technique, the verifier can detect block errors with probability $P \geq 1-\left(1-\mathrm{p}_{\mathrm{b}}\right)^{\mathrm{t}} \geq 1-((1-$ $\left.\rho)^{S}\right)^{\mathrm{Z} \cdot \omega}=1-(1-\rho)^{\mathrm{SZ} \cdot \omega}$ for a challenge with $t=n \cdot \omega$ index-coefficient pairs. In the same way, given a multi- 
cloud $\mathcal{P}=\{P i\} \in[1, c]$, the detection probability of CDDR technique has $(s z,\{\rho k, r k\} \in \mathcal{P}, \omega) \geq 1-\prod_{\mathrm{Pk} \in \mathcal{P}}((1-$ $\left.\rho \mathrm{k})^{\mathrm{s}}\right) \mathrm{r}_{\mathrm{k}} \cdot{ }^{\cdot \omega}=1-\prod_{\mathrm{Pk} \in \mathcal{P}}(1-\rho \mathrm{k})^{\mathrm{sz} \cdot \mathrm{r}_{\mathrm{k}} \cdot \omega}, \quad$ where $r_{k}$ denotes the proportion of data blocks in the $k$-th CSP, $\rho k$ denotes the probability of file corruption 2 . Exactly, we have $P=$ $1-\left(1-\frac{e}{n}\right) \cdot\left(1-\frac{e}{\mathrm{n}-1}\right) \cdots\left(1-\frac{e}{\mathrm{n}-\mathrm{t}+1}\right)$.

Since $1-\frac{e}{n} \geq 1-\frac{e}{\mathrm{n}-\mathrm{i}}$ for $i \in[0, t-1]$, we have $P=$ $1-\prod_{i=0}^{t-1}\left(1-\frac{e}{n-i}\right) \geq 1-\prod_{i=0}^{t-1}\left(1-\frac{e}{n}\right)=1-\left(1 \frac{e}{n}\right)^{t}$.

3. In terms of $\left(1-\frac{e}{n}\right)^{t} \approx\left(1-\frac{e . t}{n}\right)$, we have $P \approx 1-\left(1-\frac{e . t}{n}\right)=$ $\frac{e . t}{n}$. In the $k$-th CSP and $r k \cdot \omega$ denotes the possible number of blocks queried by the verifier in the $k$-th CSP. Furthermore, we observe the ratio of queried blocks in the total file blocks $w$ under different detection probabilities. Based on above analysis, it is easy to find that this ratio holds the

TABLE 5: The influence of $s, t$ under the different corruption probabilities $\rho$ and the different detection probabilities $P$

\begin{tabular}{|l|l|l|l|l|}
\hline $\mathcal{P}$ & $\begin{array}{l}\{0.1,0.2 \\
, 0.01\}\end{array}$ & $\begin{array}{l}\{0.01,0.0 \\
2,0.001\}\end{array}$ & $\begin{array}{l}\{0.001,0.00 \\
2,0.0001\}\end{array}$ & $\begin{array}{l}\{0.0001,0.00 \\
02,0.00001\}\end{array}$ \\
\hline$r$ & $\begin{array}{l}\{0.5,0.3 \\
0.2\}\end{array}$ & $\begin{array}{l}\{0.5,0.3, \\
0.2\}\end{array}$ & $\{0.5,0.3,0.2$ & $\{0.5,0.3,0.2\}$ \\
& $4 / 7$ & $20 / 23$ & $62 / 71$ & $71 / 202$ \\
\hline $0.8 / 3$ & $4 / 7$ & $65 / 79$ & $79 / 214$ \\
\hline $0.85 / 3$ & $5 / 8$ & $21 / 26$ & $73 / 87$ & $87 / 236$ \\
\hline $0.9 / 3$ & $6 / 10$ & $20 / 28$ & 736 & $100 / 267$ \\
\hline $0.95 / 3$ & $8 / 11$ & $29 / 31$ & $86 / 100$ & $119 / 345$ \\
\hline $0.99 / 4$ & $10 / 13$ & $31 / 39$ & $105 / 119$ & $146 / 433$ \\
\hline $\begin{array}{l}0.999 \\
/ 5\end{array}$ & $11 / 16$ & $38 / 48$ & $128 / 146$ & \\
\hline
\end{tabular}

equation $w \approx \frac{\log (1-\mathrm{P})}{\mathrm{sz} \cdot \Sigma \mathrm{Pk} \in \mathcal{P} \mathrm{rk} \cdot \log (1-\rho \mathrm{k})}$. When this probability $\rho k$ is a constant probability, the verifier can detect sever misbehavior with a certain probability $P$ by asking proof for the number of blocks $t \approx \log (1-P) s \cdot \log (1-\rho)$ for $\mathrm{DDR}$ or for $\approx \approx \frac{\log (1-\mathrm{P})}{\mathrm{s} \cdot \Sigma \mathrm{Pk} \in \mathcal{P} \mathrm{rk} \cdot \log (1-\rho \mathrm{k})} \mathrm{CDDR}$, where $t=n \cdot w$ $=\frac{\mathrm{sz} \cdot \mathrm{w}}{s}$. Note that, the value of $t$ is dependent on the total number of file blocks $n$ [2], because it is increased along with the decrease of $\rho k$ and $\log (1-\rho k)<0$ for the constant number of disrupted blocks $e$ and the larger number $n$. Another advantage of probabilistic verification based on random sampling is that it is easy to identify the tampering or forging data blocks or tags. The identification function is obvious: when the verification fails, we can choose the partial set of challenge indexes as a new challenge set, and continue to execute the verification protocol. The above search process can be repeatedly executed until the bad block is found. The complexity of such a search process is $(\log n)$.

5.3 Parameter Optimization:In the fragment structure, the number of sectors per block $s$ is an important parameter to affect the performance of storage services and audit services. Hence, we propose an optimization algorithm for the value of $\mathrm{s}$ in this section. Our results show that the optimal value can not only minimize the computation and communication overheads, but also reduce the size of extra storage, which is required to store the verification tags in CSPs. Assume $\rho$ denotes the probability of sector corruption. In the fragment structure, the choosing of $s$ is extremely important for improving the performance of the CDDR technique. Given the detection probability $P$ and the probability of sector corruption $\rho$ for multiple clouds $\mathcal{P}=\{P k\}$, the optimal value of $s$ can be computed by $\min s \in \mathbb{N}\left\{\frac{\log (1-\mathrm{P})}{\Sigma \mathrm{Pk} \in \mathcal{P} \mathrm{rk} \cdot \log (1-\rho \mathrm{k})} \cdot \frac{a}{s}+b \cdot s+c\right\}$, where $a \cdot t+b \cdot s+c$ denotes the computational cost of verification protocol in DDR technique, $a, b, c \in \mathbb{R}$, and $c$ is a constant. This conclusion can be obtained from following process: Let $s z=n \cdot s=(f) / l_{0}$. According to abovementioned results, the sampling probability holds $w \geq$ $\frac{\log (1-\mathrm{P})}{\mathrm{sz} \cdot \Sigma \mathrm{Pk} \in \mathcal{P} \text { rk } \log (1-\rho \mathrm{k})}=\frac{\log (1-\mathrm{P})}{\mathrm{n} \cdot \mathrm{s} \cdot \Sigma \mathrm{Pk} \in \mathcal{P} \mathrm{rk} \cdot \log (1-\rho \mathrm{k})}$.In order to minimize the computational cost, we have $\min s \in \mathbb{N}\{a \cdot t+$ $b \cdot s+c\}=\min s \in \mathbb{N}\{a \cdot n \cdot w+b \cdot s+c\} \geq \min s \in \mathbb{N}\{\Sigma \log (1$ -P) $P k \in \mathcal{P} r k \cdot \log (1-\rho k) a s+b \cdot s+c\}$. Whererk denotes the proportion of data blocks in the $k$-th CSP, $\rho k$ denotes the probability of file corruption in the $k$-th CSP. Since $\frac{a}{s}$ is a monotone decreasing function and $b \cdot s$ is a monotone increasing function for $s>0$, there exists an optimal value of $s \in \mathbb{N}$ in the above equation. The optimal value of $s$ is unrelated to a certain file from this conclusion if the probability $\rho$ is a constant value. For instance, we assume a multi-cloud storage involves three CSPs $\mathcal{P}=\{P 1, P 2, P 3\}$ and the probability of sector corruption is a constant value $\{\rho 1, \rho 2, \rho 3\}=\{0.01,0.02,0.001\}$. We set the detection probability $P$ with the range from 0.8 to 1 , e.g., $P=\{0.8$, $0.85,0.9,0.95,0.99$, and 0.999$\}$. For a file, the proportion of data blocks is $50 \%, 30 \%$, and $20 \%$ in three CSPs, respectively, that is, $r 1=0.5, r 2=0.3$, and $r 3=0.2$. In terms of Table 3, the computational cost of CSPs can be simplified to $t+3 s+9$. When $s$ is less than the optimal value, the computational cost decreases evidently with the increase of $s$, and then it raises when $s$ is more than the optimal value. More accurately, we show the influence of parameters, $s z \cdot w, s$, and $t$, under different detection probabilities in Table 6 . It is easy to see that computational cost rises with the increase of $P$. Moreover, we can make sure the sampling number of challenge with followingConclusion: Given the detection probability $P$, the probability of sector corruption $\rho$, and the number of 
sectors in each block $s$, the sampling number of verification protocol are a constant $t=n \cdot w$ $\geq \frac{\log (1-\mathrm{P})}{\mathrm{s} \cdot \Sigma \mathrm{Pk} \in \mathcal{P} \operatorname{rk} \cdot \log (1-\rho \mathrm{k})}$ for different files. Finally, we observe the change of sunder different $\rho$ and $P$. The experimental results are shown in Table 5. It is obvious that the optimal value of $s$ rises with increase of $P$ and with the decrease of $\rho$. We choose the optimal value of $s$ on the basis of $\rho$. settings and system requisition. For NTFS format,

TABLE 6: The influence of parameters under different detection probabilities $P(\mathcal{P}=\{\rho 1, \rho 2, \rho 3\}=\{0.01,0.02,0.001\},\{r 1$, $r 2, r 3\}=\{0.5,0.3,0.2\})$

\begin{tabular}{|l|l|l|l|l|l|l|}
\hline $\mathrm{P}$ & 0.8 & 0.85 & 0.9 & 0.95 & 0.99 & 0.999 \\
\hline$S Z \cdot W$ & 142.60 & 168.09 & 204.02 & 265.43 & 408.04 & 612.06 \\
\hline$S$ & 7 & 8 & 10 & 11 & 13 & 16 \\
\hline$t$ & 20 & 21 & 20 & 29 & 31 & 38 \\
\hline
\end{tabular}

we suggest that the value of $s$ is 200 and the size of block is 4 KBytes, which is the same as the default size of cluster when the file size is less than 16TB in NTFS. In this case, the value of $s$ ensures that the extra storage doesn't exceed $1 \%$ in storage servers.

\subsection{CDDR for Integrity Audit Services:Basedon} our CDDR technique, we introduce audit system architecture for outsourced data in multiple clouds by replacing the TTP with a third party auditor (TPA) in Figure 1. In this architecture, this architecture can be constructed into a visualization infrastructure of cloudbased storage service [1]. In Figure 3, we show an example file system [9]. HDFS' architecture is composed of NameNode and DataNode, where NameNode maps a file name to a set of indexes of blocks and DataNode indeed stores data blocks. To support our CDDR technique, the index-hash hierarchy and the metadata of NameNode should be integrated together to provide an enquiry service for the hash value $\xi_{i, k}^{(3)}$, or index-hash record $\chi i$. Based on the hash value, the clients can implement the verification protocol via CDDR services. Hence, it is easy to replace the checksum methods with the CDDR technique for anomaly detection in current HDFS. To validate the effectiveness and efficiency of our proposed approach for audit services, we have implemented a prototype of an audit system. We

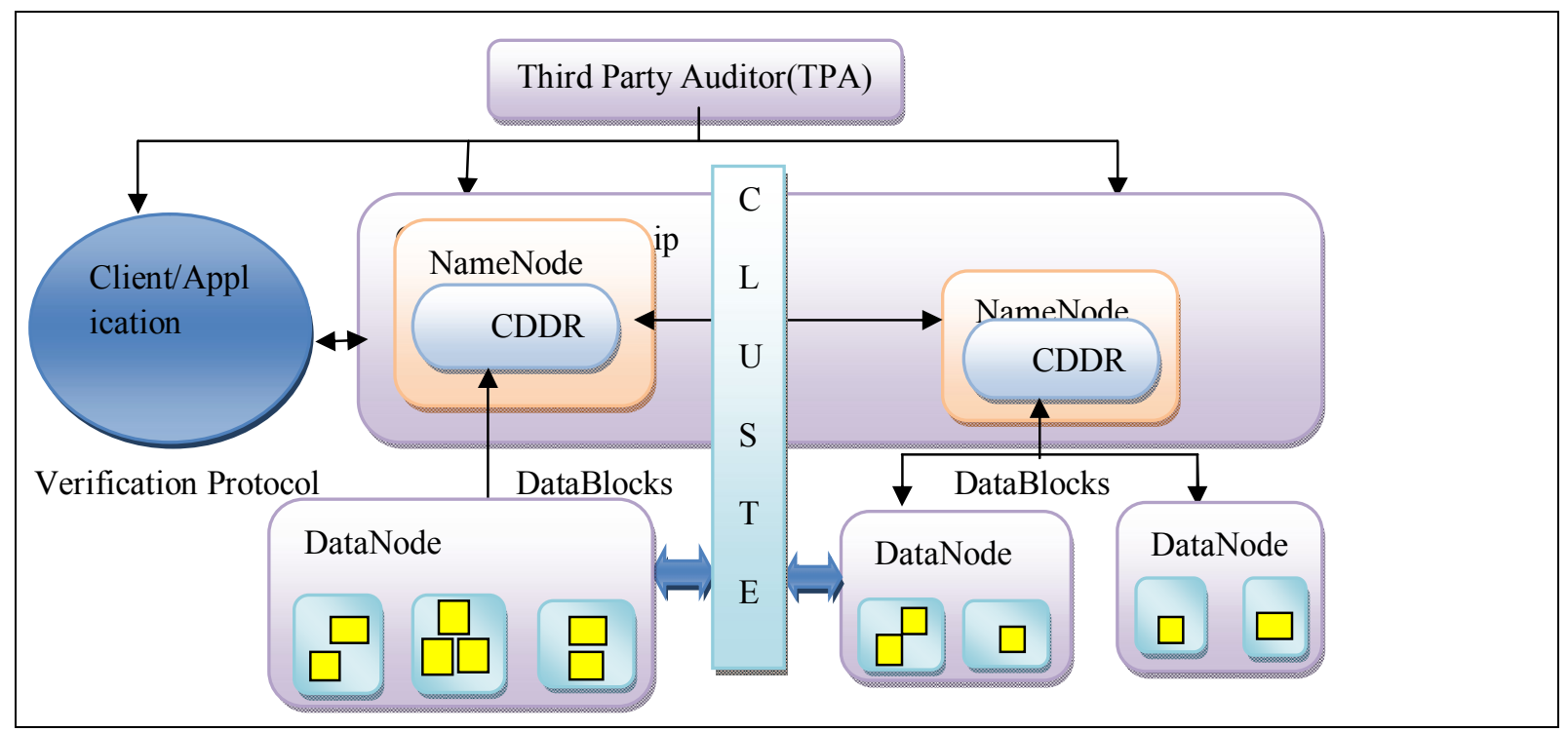

of applying our CDDR technique in Hadoop distributed filesystem (HDFS) ${ }^{4}$, with a distributed, scalable, and portable

Figure 3:Applying CDDR Technique in Hadoop distributed file system (HDFS)

two local IBM servers with two Intel Core 2 processors at 2.16 GHz and 500M RAM running Windows Server 2003.
These servers were connected via $250 \mathrm{MB} / \mathrm{sec}$ of network bandwidth. Using GMP and PBC libraries, we have 
implemented a cryptographic library upon which our technique can be constructed. This C library contains approximately 5,200 lines of codes and has been tested on both Windows and Linux platforms. The elliptic curve utilized in the experiment is a MNT curve, with base field size of 160 bits and the embedding degree 6 . The security level is chosen to be 80 bits, which means $|p|=160$. Furthermore, the proportions of data blocks in each CSP have greater influence on the computation costs of "challenge" and "response" processes.

\section{CONCLUSIONS}

We make three key contributions in this paper, first we have proposed a Cooperative DDR technique to support dynamic scalability on multiple storage servers, and second we presented the construction of an efficient DDR technique for distributed cloud storage Based on homomorphic verifiable response and hash index hierarchy. Third we also showed that our technique provided all security properties required by zeroknowledge interactive proof system, so that it can resist various attacks even if it is deployed as a public audit service in clouds. Furthermore, we optimized the probabilistic query and periodic verification to improve the audit performance. Our experiments clearly demonstrated that our approaches only introduce a small amount of computation and communication overheads. Therefore, our solution can be treated as a new candidate for data integrity verification in outsourcing data storage systems. As part of future work, we would extend our work to explore more effective CDDR constructions. For a practical point of view, we still need to address some issues about integrating our CDDR technique smoothly with existing systems, for example, how to match index structure with cluster-network model, how to match index hash hierarchy with HDFS's two-layer name space, and how to dynamically update the CDDR parameters according to HDFS' specific requirements. Finally, it is still a challenging problem for the generation of tags with the length irrelevant to the size of data blocks. We would explore such an issue to provide the support of variable-length block verification.

\section{ACKNOWLEDGEMENT}

The work on "Cooperative demonstrable data retention for integrity verification in multi-cloud Storage" was supported by many members. We are highly obliged and thankful to almighty who has provided us with the opportunity to thank all the kins who stood by us in the process of working on this project. First of all we would like to thank Mr. Shubham Srivastavaour project guide andwho was of utmost help to us throughout the proceedings. Further, we would like to thank Head of Computer Science department of Institute of Technology and Management Mr. Rajeev Ranjan Kumar Tripathi, who has been a great help and an inspiration in carrying out the present work successfully. So it's our pleasure to present a cordial thanks to him.

\section{REFERENCES}

[1]. G. Ateniese, R. C. Burns, R. Curtmola, J. Herring, L. Kissner, Z. N. J. Peterson, and D. X. Song, "Provable data possession at untrusted stores," in ACM Conference on Computer and Communications Security, P. Ning, S. D. C. di Vimercati, and P. F. Syverson, Eds. ACM, 2007, pp. 598-609.

[2]. A. Juels and B. S. K. Jr., "Pors: proofs of retrievability for large files," in ACMConference on Computer and Communications Security, P. Ning, S. D. C. di Vimercati, and P. F. Syverson, Eds. ACM, 2007, pp. 584-597.

[3]. B. Sotomayor, R. S. Montero, I. M. Llorente, and I. T. Foster, "Virtual infrastructure management in private and hybrid clouds," IEEE Internet omputing, vol. 13, no. 5, pp. 14-22, 2009.

[4]. G. Ateniese, R. D. Pietro, L. V. Mancini, and G. Tsudik, "Scalable and efficient provable data possession," in Proceedings of the 4th international conference on Security and privacy in communi cation netowrks, SecureComm, 2008, pp. 1-10.

[5]. C. C. Erway, A. K"upc, "u, C. Papamanthou, and R. Tamassia, "Dynamic provable data possession," in ACM Conference on Computer and Communications Security, E. Al-Shaer, S. Jha, and A. D. Keromytis, Eds. ACM, 2009, pp. 213-222.

[6] H. Shacham and B. Waters, "Compact proofs of retrievability," in ASIACRYPT, ser. Lecture Notes in Computer Science, J. Pieprzyk, Ed., vol. 5350. Springer, 2008, pp. 90-107.

[7] Q. Wang, C.Wang, J. Li, K. Ren, and W. Lou, "Enabling public verifiability and data dynamics for storage security in cloud computing," in ESORICS, ser. Lecture Notes in ComputerScience, M. Backes and P. Ning, Eds., vol. 5789. Springer,2009, pp. 355370.

[8] Y. Zhu, H. Wang, Z. Hu, G.-J. Ahn, H. Hu, and S. S. Yau, "Dynamicaudit services for integrity verification of outsourcedstorages in clouds," in SAC, W. C. Chu, W. E. Wong, M. J.Palakal, and C.-C. Hung, Eds. ACM, 2011, pp. 1550-1557.

[9] K. D. Bowers, A. Juels, and A. Oprea, "Hail: a highavailabilityand integrity layer for cloud storage," in ACM Conference onComputer and Communications Security, E. Al-Shaer, S. Jha, andA. D. Keromytis, Eds. ACM, 2009, pp. 187-198. 
International Journal of Computer Applications Technology and Research Volume 2- Issue 2, 195 - 207, 2013, ISSN: 2319-8656

[10] Y. Dodis, S. P. Vadhan, and D. Wichs, "Proofs of retrievabilityvia hardness amplification," in TCC, ser. Lecture Notes inComputer Science, O. Reingold, Ed., vol. 5444. Springer, 2009,pp. 109-127.

[11] L. Fortnow, J. Rompel, and M. Sipser, "On the power of multiprover interactive protocols," in Theoretical Computer Science, 1988, pp. 156-161.

[12] Y. Zhu, H. Hu, G.-J. Ahn, Y. Han, and S. Chen, "Collaborative integrity verification in hybrid clouds," in IEEE Conference on the 7th International Conference on Collaborative Computing: Networking, Applications and Worksharing, CollaborateCom, Orlando, Florida, USA, October 15-18, 2011, pp. 197206.

[13] M. Armbrust, A. Fox, R. Griffith, A. D. Joseph, R. H. Katz, A. Konwinski, G. Lee, D. A. Patterson, A. Rabkin, I. Stoica, and M. Zaharia, "Above the clouds: A berkeley view of cloud computing". 